\section{Electrocauterio y broncoscopia flexible como primera medida en la obstrucción central de la vía aérea y hemoptisis asociada}

\author{
ALFREDO JALILIE ${ }^{1}$, JUAN CARLOS CARVAJAL ${ }^{1}$, \\ RODRIGO APARICIO ${ }^{2}$, MANUEL MENESES ${ }^{3}$
}

\section{Electrocautery and bronchoscopy as a first step for the management of central airway obstruction and associated hemoptysis}

Background: Central airway obstruction caused by malignant or benign lesions, associated in some cases with hemoptysis, is a condition with high morbidity and mortality. The use of electrocautery by flexible bronchoscopy is an initial treatment option with immediate improvement of obstruction symptoms. It is as effective as Nd: YAG laser. Aim: To describe the usefulness of electrocautery in the management of central obstruction of the airway and hemoptysis. Material and Methods: A retrospective, descriptive study of patients referred for management of central airway obstruction or associated hemoptysis. Diagnoses, symptoms (dyspnea, cough, and hemoptysis) and radiology before and after the procedures were analyzed. Results: Eighteen patients aged $59 \pm 12$ years (66\% males) were evaluated, registering 25 endoscopic procedures. Three conditions were found: partial or complete airway obstruction, hemoptysis and post lung transplant bronchial stenosis. Seventy two percent presented with dyspnea, 61\% with cough and 33\% with hemoptysis. Sixty six percent of patients had airway obstruction caused by malignant metastatic lesions. After electrocautery, 17 patients (94.4\%) improved their symptoms and achieved complete airway clearing. Three patients had significant bronchial stenosis after lung transplant achieving subsequent clearing after electrocautery. Conclusions: Electrocautery during flexible bronchoscopy is an effective and safe procedure for the management of central airway obstruction and associated hemoptysis.

(Rev Med Chile 2016; 144: 1417-1423)

Key words: Bronchial Neoplasms; Bronchoscopy; Electrocoagulation; Hemoptysis; Lung Transplantation.

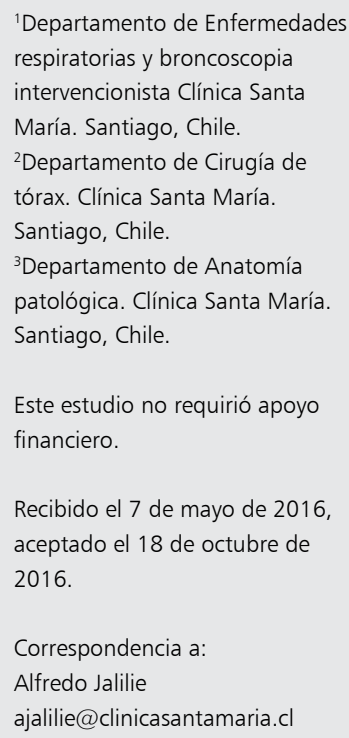

Este estudio no requirió apoyo financiero.

Recibido el 7 de mayo de 2016 aceptado el 18 de octubre de 2016.

Correspondencia a:

Alfredo Jalilie

ajalilie@clinicasantamaria.cl

L

a permeabilidad de la vía aérea puede verse comprometida por una serie de patologías que representan un riesgo para la vida del paciente. Existen reportes en la literatura de obstrucción endoluminal traqueo-bronquial tanto por tumores malignos, sangre o por patología benigna, demostrándose una importante mor- bi-mortalidad asociada a estas condiciones ${ }^{1}$. El uso de la broncoscopia intervencionista se ha incrementado en las dos últimas décadas, empleándose tratamientos curativos o paliativos mediante el uso separado o combinado de técnicas de remoción de tejido por desbridamiento, dilatación, terapias ablativas y/o colocación de stent traqueo-bron- 
quiales $^{2}$. Con respecto a las terapias ablativas con efecto inmediato tanto el $\mathrm{Nd}$ : Yttrium aluminium garnet (YAG) láser, la coagulación por Argón Plasma (AP) y la electrocauterización, pueden usarse directamente vaporizando el tejido y con mayor frecuencia, coagulándolo antes de su desbridamiento mecánico ${ }^{2}$. El Nd: YAG láser tiene una excelente penetración en los tejidos con un potente poder de ablación mientras que AP se limita a manejo de lesiones superficiales de 1 a $2 \mathrm{~mm}$. Múltiples estudios han demostrado la utilidad de Nd: YAG láser en el manejo de la obstrucción central de la vía área en términos clínicos y radiológicos, sin embargo, su alto costo y poca disponibilidad en muchos centros lo hacen una técnica poco utilizada ${ }^{3,5,6}$.

El electrocauterio es una interesante opción de terapia ablativa mediante flujos de energía eléctrica que permiten un efecto electrolítico y térmico, facilitando la resección y coagulación de los tejidos. Fue en 1932 cuando se describió por primera vez su utilidad en el árbol traqueobronquial, tomando mayor fuerza después de los años ochenta ${ }^{1-3}$.

Su coste está muy por debajo del Nd YAG láser y por lo tanto, existe mayor disponibilidad. Sin embargo, la experiencia en general con el manejo de la permeabilización de la vía aérea no ha sido muy difundida, y para muchos neumólogos sigue siendo una técnica poco conocida ${ }^{4}$.

El presente trabajo tiene por objetivo mostrar la utilidad del electrocauterio mediante la experiencia lograda en Clínica Santa María en el manejo de la permeabilización de la vía aérea y alivio de síntomas relacionados, en pacientes con patología respiratoria maligna, hemoptisis asociada y patología benigna.

\section{Materiales y Métodos}

Se realizó un estudio retrospectivo, de tipo descriptivo, de pacientes remitidos para manejo de la vía aérea por obstrucción central y/o hemoptisis asociada, en los que se realizó electrocauterización endobronquial entre enero de 2012 y marzo de 2016 en la Unidad de Broncoscopia de Clínica Santa María, Santiago de Chile. Se incluyeron procedimientos ambulatorios y hospitalizados. Todos los pacientes firmaron consentimiento informado según las normas del centro y la unidad de broncoscopia antes de la ejecución del procedimiento.
El estudio de investigación fue aprobado por el comité de ética científico de clínica Santa María, Santiago de Chile.

La electrocauterización se llevó a cabo con un equipo Electrosurgical Generator (ESG-100) Olympus, incluyendo sonda y asa de $10 \mathrm{~mm}$ dedicadas, de la misma marca. La remoción del tejido tratado se realizó con fórceps Alligator, Olympus para biopsias. El uso de la potencia eléctrica se ajustó según las necesidades del caso y bajo las recomendaciones del fabricante, utilizándose entre 30 a 60 watts para cada caso. Los procedimientos fueron realizados en la unidad de cirugía endoscópica de Clínica Santa María, Santiago de Chile por un solo endoscopista, bajo sedación consciente utilizando Midazolam y Fentanilo, con apoyo con oxígenoterapia $\left(\mathrm{O}_{2}\right)$ con fracción inspiratoria de 0,6 y suspendiendo transitoriamente la administración de $\mathrm{O}_{2}$ al momento de la electrofulguración.

Se hizo monitorización del paciente con electrocardiografía continua, saturometría y presión arterial. Se utilizó en todos los casos un videobroncoscopio terapéutico Olympus BT-180 Japan. En caso de sangrado durante el procedimiento se empleo instilaciones de solución de Adrenalina 1 $\mathrm{ml}$ de 1:10.000 y solución salina al 0,9\% fría.

Todos los pacientes tuvieron por lo menos un control broncoscópico al mes posterior al manejo con electrocauterio.

\section{Datos recolectados}

Utilizando el sistema de ficha electrónica de la institución se pudo recolectar información para el estudio.

- Datos demográficos.

- Diagnósticos e indicaciones de broncoscopia.

- Estudio histológico final en caso de lesiones endobronquiales.

- Signos y síntomas pre y post procedimiento broncoscópico: disnea, tos y hemoptisis.

- Imágenes radiográficas pre y post procedimiento.

Se evaluó respuesta clínica y/o radiológica según el caso.

Se registraron complicaciones asociadas al procedimiento.

La respuesta clínica fue evaluada de forma subjetiva por el médico broncoscopista y consideró: alivio de síntomas como disnea, tos persistente y control inmediato de hemoptisis. 
Se consideró como respuesta radiológica la expansión pulmonar radiológica parcial o completa en caso de atelectasia masiva o lobar en la radiografía de tórax.

\section{Resultados}

Dieciocho pacientes fueron sometidos a electrocoagulación endobronquial con un total de 25 procedimientos. Los pacientes fueron referidos al procedimiento por sintomatología respiratoria y/o hemoptisis. La edad promedio de los pacientes fue de 59,09 años (rango 17-80 años, DE $\pm 12,5$ ). Doce pacientes $(66,7 \%)$ fueron de género masculino.

Se clasificaron tres condiciones: 1) Obstrucción bronquial parcial o completa en 15 pacientes; 2) Hemoptisis por lesión bronquial obstructiva en 6 pacientes; 3 ) Estenosis bronquial post trasplante de pulmón con deterioro clínico en 3 pacientes.

Doce pacientes tuvieron diagnóstico de neoplásica maligna metastásica obstructiva (66\%). siendo más frecuente la de origen renal. Tres pacientes tuvieron diagnóstico de tumor carcinoide típico bronquial. Tres pacientes correspondían a trasplantados de pulmón, los que presentaron estenosis mayor al 90\% del lumen bronquial, post trasplante: dos pacientes con importante disnea en el contexto de síndrome de desvanecimiento del bronquio intermediario que representa una complicación grave secundaria a isquemia bronquial post trasplante y uno tuvo estenosis de la anastomosis bronquial izquierda (Tabla 1). Todos los pacientes presentaron síntomas al momento de la consulta: disnea (72\%), tos $(61 \%)$ y/o hemoptisis $(33 \%)$. Cinco pacientes presentaron obstrucción bronquial completa de los bronquios fuentes principales: dos por metástasis de cáncer renal, uno por cáncer pulmonar, uno por tumor carcinoide y uno por metástasis de cáncer de colon. Tres de ellos, presentaron atelectasia completa del pulmón correspondiente al bronquio fuente afectado (Tabla 2).

\section{Tabla 1. Indicaciones de electrocauterización}

\begin{tabular}{|lc|}
\hline & $\begin{array}{c}\text { Número } \\
\text { pacientes }\end{array}$ \\
\hline 1. Obstrucción bronquial parcial o completa & $15(83,3 \%)$ \\
- Metástasis endobronquial por cáncer renal & 6 \\
- Metástasis endobronquial por cáncer de colon & 2 \\
- Cáncer pulmonar con compromiso & \\
endobronquial & 3 \\
- Tumores Carcinoides endobronquiales & 3 \\
- Metástasis endobronquial por timoma maligno & 1 \\
2. Hemoptisis asociada a lesión bronquial & $6(33,3 \%)$ \\
obstructiva & \\
- Metástasis endobronquiales por cáncer renal & 3 \\
- Metástasis endobronquial por cáncer de colon & 1 \\
- Cáncer pulmonar con compromiso endoluminal & 1 \\
- Metástasis endobronquial por timoma maligno & 1 \\
3. Estenosis endobronquial post trasplante & $3(18,7 \%)$ \\
de pulmón & \\
- Desvanecimiento bronquio intermediario & 2 \\
- Estenosis de anastomosis izquierda & 1 \\
\hline
\end{tabular}

Tabla 2. Bronquios comprometidos por neoplasia

\begin{tabular}{|c|c|c|}
\hline & $\mathbf{n}$ & Tipo de neoplasia \\
\hline $\begin{array}{l}\text { Bronquio principal izquierdo } \\
\text { - Oclusión completa } \\
\text { - Oclusión parcial }\end{array}$ & $\begin{array}{l}3 \\
1\end{array}$ & $\begin{array}{l}\text { Tumor carcinoide y cáncer renal (2) } \\
\text { Tumor carcinoide }\end{array}$ \\
\hline $\begin{array}{l}\text { Bronquio principal derecho } \\
\text { - Oclusión completa } \\
\text { - Oclusión parcial }\end{array}$ & $\begin{array}{l}2 \\
2\end{array}$ & $\begin{array}{l}\text { Cáncer de colon, cáncer pulmonar } \\
\text { Cáncer renal ( } 2 \text { ) }\end{array}$ \\
\hline $\begin{array}{l}\text { Bronquio Intermediario } \\
\text { - Oclusión parcial }\end{array}$ & 4 & Tumor carcinoide, cáncer de Colon, cáncer renal (2) \\
\hline $\begin{array}{l}\text { Bronquio lobar superior derecho } \\
\text { - Oclusión completa } \\
\text { - Oclusión parcial }\end{array}$ & $\begin{array}{l}1 \\
1\end{array}$ & $\begin{array}{l}\text { Timoma maligno } \\
\text { Cáncer pulmonar }\end{array}$ \\
\hline $\begin{array}{l}\text { Bronquio lobar inferior izquierdo } \\
\text { - Obstrucción completa }\end{array}$ & 1 & Cáncer pulmomar \\
\hline
\end{tabular}



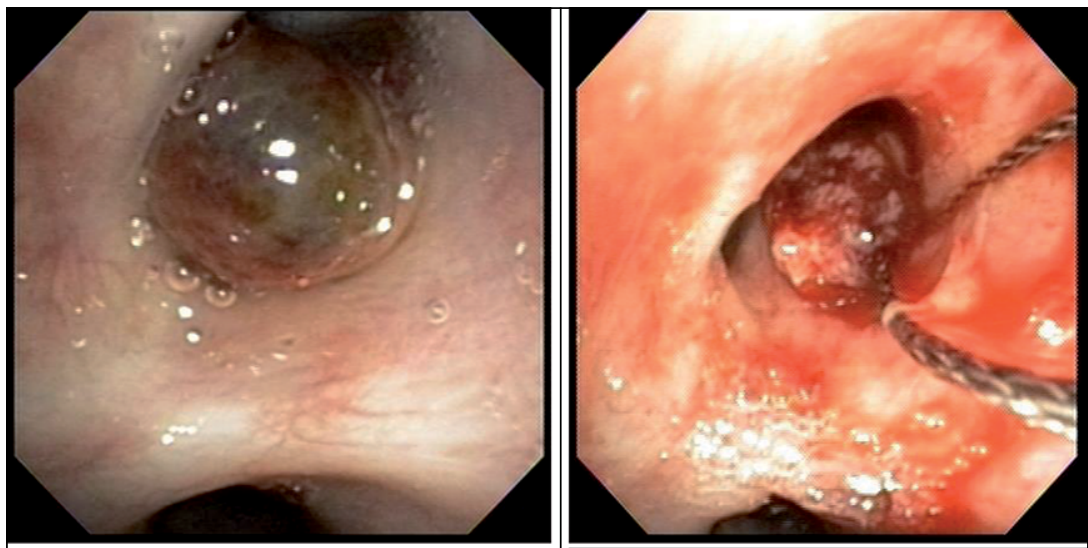

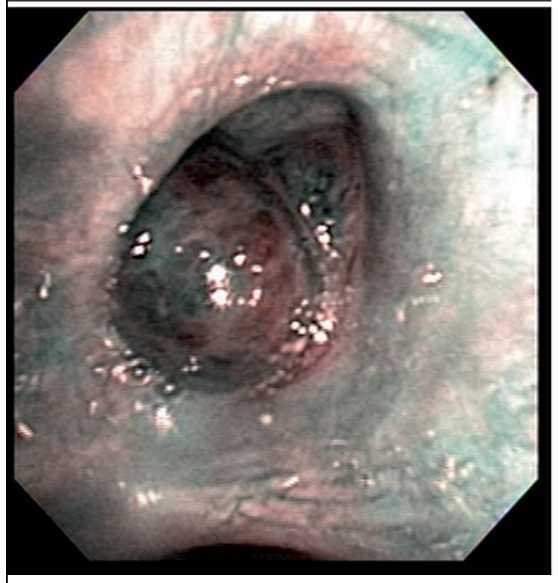

A

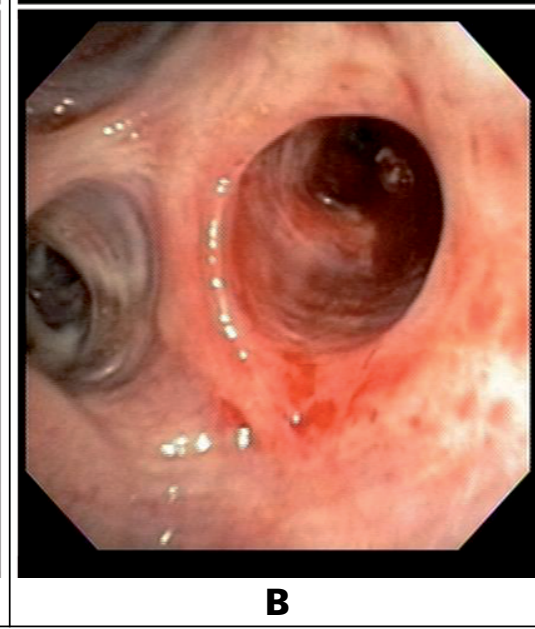

Figura 1. A. Metástasis de timoma maligno con oclusión completa del bronquio lobar superior derecho. B. Permeabilización del bronquio lobar superior derecho después del uso de electrocauterio endobronquial.

\section{Resultados después de la electrocauterización}

Posterior al procedimiento diecisiete pacientes $(94,4 \%)$ experimentaron mejoría en sus síntomas de tos, disnea y/o hemoptisis en forma inmediata según evaluación subjetiva por el médico broncoscopista, permeabilizándose completamente el lumen bronquial (Figura 1).

En sólo un paciente no fue posible la permeabilización satisfactoria por la extensión de la lesión endobronquial que correspondió a una metástasis por cáncer pulmonar.

Ocho pacientes se presentaron con obstrucción completa de algún bronquio. De ellos, 3 tuvieron atelectasia pulmonar total. Luego del procedimiento con electrocauterio, se observó expansión completa del pulmón afectado en la radiografía de tórax en dos pacientes y en uno en forma parcial.

Tres pacientes se presentaron con tumores carcinoides típicos. Dos de ellos con lesiones obstructivas parciales que pudieron resecarse por completo, con un seguimiento de hasta 10 meses sin recidiva local (Figura 2). El otro paciente se presentó con atelectasia masiva de pulmón izquierdo por tumor oclusivo del bronquio fuente principal. El electrocauterio pudo repermeabilizar hasta un $85 \%$ del lumen bronquial, resolviendo la disnea, tos y la atelectasia.

Respecto a los pacientes trasplantados de pulmón, la electrocauterización permitió permeabilizar los bronquios afectados, para luego acceder a la dilatación endobronquial con balón, con mejoría significativa de la disnea y de la tos. Dos de ellos requirieron más de un procedimiento con electrocauterio (Figura 3).

El control broncoscópico al primer mes posterior del procedimiento con electrocauterio demostró persistencia de la permeabilidad bronquial en 14 de 15 pacientes con lesiones endobronquiales resecadas, sin evidencia de sangrado en la zona intervenida. Los 3 pacientes con estenosis bron- 

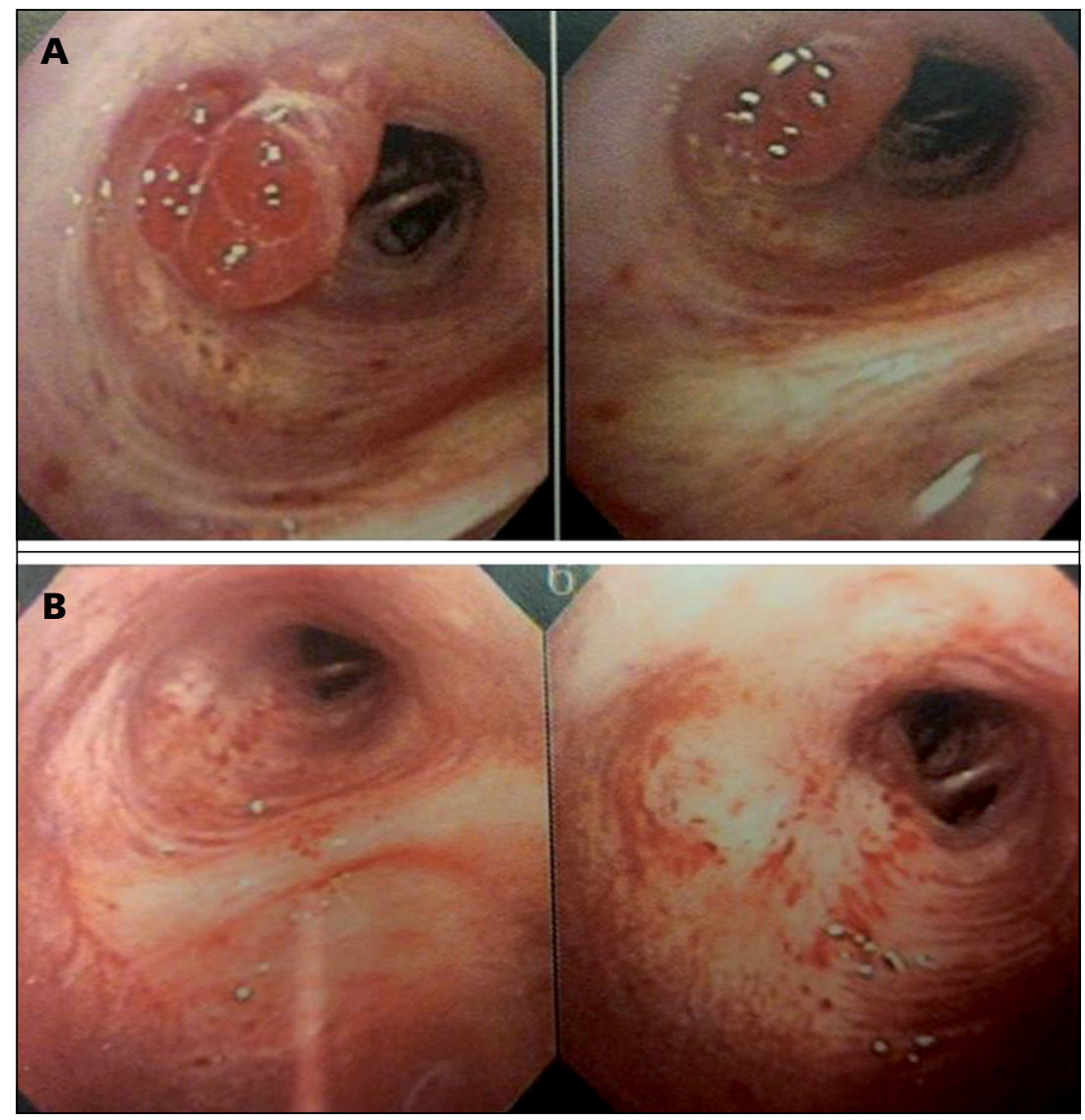

Figura 2. A. Tumor carcinoide en bronquio intermediario. B. Tres meses posteriores a la electrocauterización.

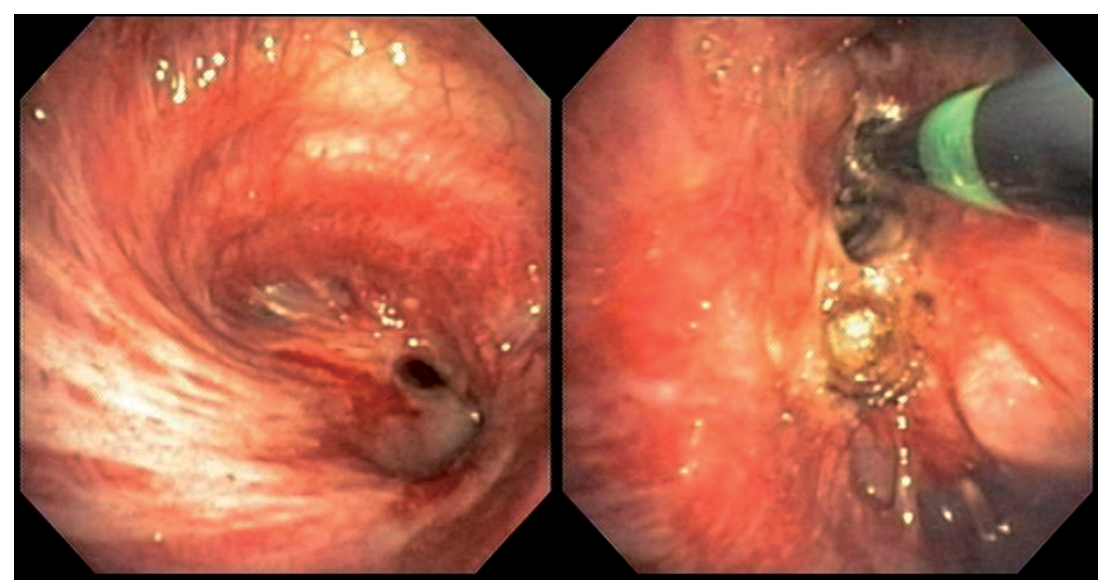

Figura 3. Síndrome de desvanecimiento del bronquio intermediario post trasplante pulmonar derecho. Manejo con electrocauterio endobronquial.

quiales post trasplantes pulmonar mantuvieron permeables los bronquios tratados.

No se registraron complicaciones mayores en ningún paciente posterior a los 25 procedimientos realizados en los 18 pacientes analizados.

\section{Discusión}

Si bien el uso de electrocauterio para el manejo de la obstrucción traqueo bronquial y hemoptisis ha tenido amplia difusión en las dos últimas dé- 
cadas, la cantidad de información en la literatura médica sobre su utilidad e impacto en el manejo inmediato de los síntomas del paciente, ha sido limitada a pocos estudios con series de casos $^{7}$.

Hay datos suficientes que demuestran la utilidad del Nd: YAG láser en el manejo de la oclusión central de la vía aérea por neoplasia o procesos benignos, sin embargo, se trata de un método de alto costo y no disponible en la mayoría de centros hospitalarios. No ocurre así con los equipos de electrocauterio que son de más bajo costo y con gran disponibilidad hospitalaria. A pesar de ello, la experiencia está limitada por la subestimación y desconocimiento en su utilidad. Se trata de un método ablativo de rápida acción, donde el paciente puede manejarse en forma ambulatoria con alivió inmediato de los síntomas asociados a la obstrucción bronquial o hemoptisis ${ }^{3}$.

Sutejeda et $\mathrm{al}^{9}$ reportó 17 pacientes con obstrucción central tratada con electrocauterio endobronquial vía broncoscopia flexible. Quince de los 17 pacientes tuvieron una inmediata permeabilización de los lúmenes bronquiales afectados, alcanzando en la mayoría de los casos un calibre bronquial por sobre el $75 \%$, con un excelente control de la hemoptisis. No hubo complicaciones graves en esa serie estudiada.

Caulter y $\mathrm{Metha}^{8}$, por otro lado, publicaron una serie de 38 pacientes que fueron sometidos a manejo de vía aérea por obstrucción central usando broncoscopia flexible y electrocauterio con una tasa de éxito del $89 \%$, señalándose que en una gran cantidad de pacientes, el electracauterio pudo reemplazar a Nd: YAG láser en el manejo de esta condición.

De esta forma y según la literatura no hay datos que sugieran superioridad en efectividad o seguridad del Nd: YAG láser sobre el electrocauterio en el manejo de obstrucción central de la vía aérea.

Wahidi et $\mathrm{a}^{10}$ en un importante centro de broncoscopia intervencional en Duke, mostraron la gran utilidad del electrocauterio como un método de ablación primaria para obstrucción de la vía aérea por causas malignas y benignas. Este estudio retrospectivo, analizó 94 pacientes en los que se realizaron 117 procedimientos. Casi el $40 \%$ de los casos se hicieron a través de broncoscopia flexible obteniendo mejoría clínica en más del 70\% de los pacientes intervenidos. Sólo se reportó una complicación mayor $(0,8 \%)$ que consistió en un proceso aspirativo relacionado a la inducción anestésica. Esto datos apoyan nuestros resultados tanto en la efectividad y seguridad del procedimiento.

En nuestra casuística, tuvimos 3 pacientes con tumores carcinoides típicos. Como señalamos en los resultados, dos de ellos que se presentaron con obstrucción parcial de la vía aérea, pudieron resecarse las lesiones por completo mediante broncoscopia flexible y electrocauterio, haciéndose ablación del tejido cicatricial residual, en los seguimientos de hasta 9 meses, sin evidenciarse recidiva. La decisión del manejo endoscópico y no quirúrgico de dichas lesiones fue evaluado por el médico tratante y quedó en observación según la evolución en el tiempo en los seguimientos broncoscópicos. Si bien se trata de dos casos, hay que tener en cuenta el potencial curativo de la técnica en situaciones especiales. Existe muy poca literatura reportada a este respecto como para emitir conclusiones ${ }^{12}$.

En relación al manejo de las estenosis benignas de la vía aérea como las referidas en los pacientes con trasplante pulmonar de nuestra serie, existe experiencia internacional en el manejo inicial con electrocauterio y broncoscopia flexible ${ }^{11}$. La apertura de estenosis severas, como en el síndrome de desvanecimiento bronquial, con cortes radiales de la zona afectada es fundamental para complementar el tratamiento endoluminal con otras técnicas como la dilatación con balón o instalación de stents endobronquiales ${ }^{11}$.

Como se demuestra en nuestra experiencia, al igual que en la literatura médica, el uso de electrocauterio mediante broncoscopia flexible con sedación consciente, es posible, segura y efectiva. No reportamos complicaciones asociadas al procedimiento, sin embargo, por el mecanismo de acción que presenta la electrocauterización, se ha reportados daño superficial en la vía aérea, como también potencial daño cartilaginoso ${ }^{4}$. Otras complicaciones mayores reportadas en otros estudios fueron la perforación bronquial y el incendio de la vía aérea durante el impulso eléctrico al contacto con fracción inspirada alta de $\mathrm{O}_{2}$, pudiéndose minimizar este último hecho con la suspensión transitoria del aporte de $\mathrm{O}_{2}^{2}$.

Las limitaciones metodológicas que pueda tener nuestro estudio se explican por el carácter retrospectivo del análisis y la evaluación subjetiva de la respuesta sintomática, no obstante, todos los pacientes tratados, fueron manejados y observados 
por el mismo endoscopista (AJ), con los mismos criterios de manejo minimizando la variabilidad en la técnica y experiencia, dando fuerza a los resultados en términos de efectividad y seguridad del procedimiento.

\section{Conclusiones}

Evaluada nuestra experiencia y comparada con los datos reportados en la literatura médica, podemos concluir que el electrocauterio por broncoscopia flexible es un procedimiento efectivo y seguro en el manejo inicial de la obstrucción de la vía aérea central maligna o benigna y en hemoptisis por lesiones bronquiales.

\section{Referencias}

1. Ernst A, Feller-Kopman D, Becker H, Mehta A. Central Airway Obstruction. Am J Respir Crit Care Med 2004; 169: 1278-97.

2. Beamis J. Interventional pulmonology techniques for treating malignat large airway obstruction: an update. Curr Opi Pulm Med 2005; 11: 292-5.

3. Bolliger CT, Sutedja TG, Strausz J, Freitag L. Therapeutic bronchoscopy with inmediate effect: Laser, electrocautery, argon plasma coagulations. Chest 2006: 130: 1419-23.

4. Boxem T, Muller M, Venmans B. Nd-YAG laser vs Bronchoscopic electrocautery for palliation of syntomatic airways obstruction: a cost-effectiveness study. Chest 1991; 116: 1108-12.
5. Sachdeva A, Pickering E, Lee H. From electrocautery, balloon dilatation, neodymiumdoped: yttrium-aluminum-garnet (Nd:YAG) laser to argon plasma coagulation and cryotherapy. J Thorac Dis 2015; 7 (S4): 363-79.

6. Mertoglu A, Erbaycu A, Guclu S, Ozsoz A, Ozacar R. Therapeutic bronchoscopy in patientes with sintomatic airway lesions Argon Plasma coagulation/Electrocautery. International journal of Haematology and Oncology 2015; 3 (25): 186-94.

7. Sindhwani G, Rawat J, Keserwani V. Role of endobronchial electrocautery in management of neoplastic central airways obstruction: Initial experience with seven cases. Indian J Chest Dis Allied Sci 2012; 54 (3): 165-8.

8. Couler T, Mehta A. The heat is on: impact of endobronchial electrosurgery on the need for Nd-YAG laser photoresection. Chest 2000; 118: 516-21.

9. Sutejeda G, Van Kristensen K, Schramel F, Postmus P. Fibreoptic bronchoscopy electrosurgery under local anaesthesia for rapid palliation in patients with central airway malignancies: a preliminary report. Thorax 1994; 49: 1243-6.

10. Wahidi M, Unroe M, Adlakha N, Beyea M, Shofer S. The use of electrocautery as the primary ablation modality for malignant and benign airway obstruction. J Thoracic Oncol 2011; 6: 1516-20.

11. Santacruz JF, Mehta A. Airway complications and management after lung trasplantation. Proc Am Thorac Soc 2009; 6: 79-93.

12. Hadda V, Madam K, Mohan A, Kalai U, Guleria R. Case Report: Successful Flexible Bronchoscopic Management of Dynamic Central Airway Obstruction by a Large Tracheal Carcinoid Tumor. Case Reports in Pulmonology 2014; 1: 1-4. 